\title{
A Phase II Study on Safety and Efficacy of High-dose N-ACEtylcysteine in Patients with Cystic Fibrosis
}

\author{
N. Dauletbaev ${ }^{1}$, P. Fischer ${ }^{1}$, B. Aulbach ${ }^{1}$, J. Gross ${ }^{2}$, W. Kusche ${ }^{3}$, U. Thyroff-Friesinger ${ }^{2}$, T. O. F. Wagner ${ }^{1}$, \\ J. Bargon 1,4 \\ ${ }^{1}$ University Hospital, Frankfurt/Main, ${ }^{2}$ Hexal AG, Holzkirchen, ${ }^{3}$ A.CRO Clinical Research Services GmbH, Wiesbaden, \\ ${ }^{4}$ Hospital St. Elisabeth, Frankfurt/Main, Germany
}

\begin{abstract}
Objective: We conducted a single-centre, randomised, double-blinded, placebo-controlled phase II clinical study to test safety and efficacy of a 12-week therapy with low-dose (700 mg/daily) or high-dose (2800 $\mathrm{mg} /$ daily) of NAC.

Methods: Twenty-one patients $(\Delta \mathrm{F} 508$ homo/heterozygous, $\mathrm{FEV}_{1}>40 \%$ pred.) were included in the study. After a 3-weeks placebo run-in phase, 11 patients received low-dose NAC, and 10 patients received high-dose NAC. Outcomes included safety and clinical parameters, inflammatory (total leukocyte numbers, cell differentials, TNF- $\alpha$, IL-8) measures in induced sputum, and concentrations of extracellular glutathione in induced sputum and blood.

Results: High-dose NAC was a well-tolerated and safe medication. High-dose NAC did not alter clinical or inflammatory parameters. However, extracellular glutathione in induced sputum tended to increase on high-dose NAC.

Conclusions: High-dose NAC is a well-tolerated and safe medication for a prolonged therapy of patients with CF with a potential to increase extracellular glutathione in CF airways.
\end{abstract}

Key words: Cystic fibrosis, N-acetylcysteine, induced sputum, glutathione, inflammation

\section{INTRODUCTION}

Cystic fibrosis (CF) lung disease is the main cause of morbidity and mortality in patients with CF. Excessive neutrophil-dominated inflammation in airways is one of the hallmarks of CF lung disease. This uncontrolled inflammation is believed to lead to lung damage and dysfunction. There is a clear need for new anti-inflammatory medications in CF. The use of existing anti-inflammatory therapies, such as oral corticosteroids [1, 2] or high-dose ibuprofen [3, 4], is limited because of extensive adverse events [5] or concerns thereof [6].

Antioxidant drugs, such as $\mathrm{N}$-acetylcysteine (NAC), have attracted attention recently as potential therapies for CF. The rationale to employ e.g. NAC is based on the premise that CF airways are overexposed to oxidants derived from bacteria [7,8] or activated neutrophils [9]. Overexposure to oxidants, i.e. oxidative stress, is a known amplifier of inflammation. There- fore, antioxidant drugs may be useful to control both oxidative stress and excessive inflammation in CF airways.

While the safety and clinical efficacy of corticosteroids and high-dose ibuprofen have been thoroughly tested in clinical studies, NAC has not been studied as extensively. To date, only one short-term, open, uncontrolled, phase I study on NAC in CF has been published [10]. These authors have tested NAC administered for 4 weeks.

We have designed and conducted a single-centre, randomised, double-blinded, placebo-controlled phase II clinical study. We tested safety of a 12 -week therapy with low-dose $(700 \mathrm{mg} /$ daily) and high-dose (2800 $\mathrm{mg} /$ daily) of NAC, as well as its effects on clinical parameters, concentrations of extracellular glutathione in induced sputum and blood, and inflammatory markers in induced sputum of patients with $\mathrm{CF}$.

\section{Methods \\ PATIENTS}

The study was conducted between January 2000 and September 2001 at the adult out-patient clinic of the Hospital of Johann-Wolfgang-Goethe University, Frankfurt/Main, Germany. Eligible patients were older than 16 yrs and had an established diagnosis of CF (repeatedly positive sweat tests, homo- or heterozygous for $\Delta$ F508). Patients had stable disease within the last four weeks before enrolment, and FEV1 > 40\% pred. Patients were not to be included if they had recent (i.e. within the last four weeks) exacerbation of CF lung disease, recent use of oral corticosteroids or parenteral antibiotic therapy, history of haemoptysis, known hypersensitivity to NAC or inactive ingredients of the study medication (lactose, sodium cyclamate, saccharin sodium, polyethylene glycol, sodium carbonate, sodium bicarbonate, citric acid), history of severe drug-related allergy, and clinically significant liver impairment (AST/ GOT $\geq 50 \mathrm{IU} / \mathrm{L}$ ). Informed consents were obtained from all patients. The study protocol was approved by the local Human Ethics Board and conducted according to GCP guidelines.

\section{Study Design and Medications}

The study was conducted as a single-centre, randomised, double-blind, placebo-controlled, parallel- 


\section{A. Study flow chart}

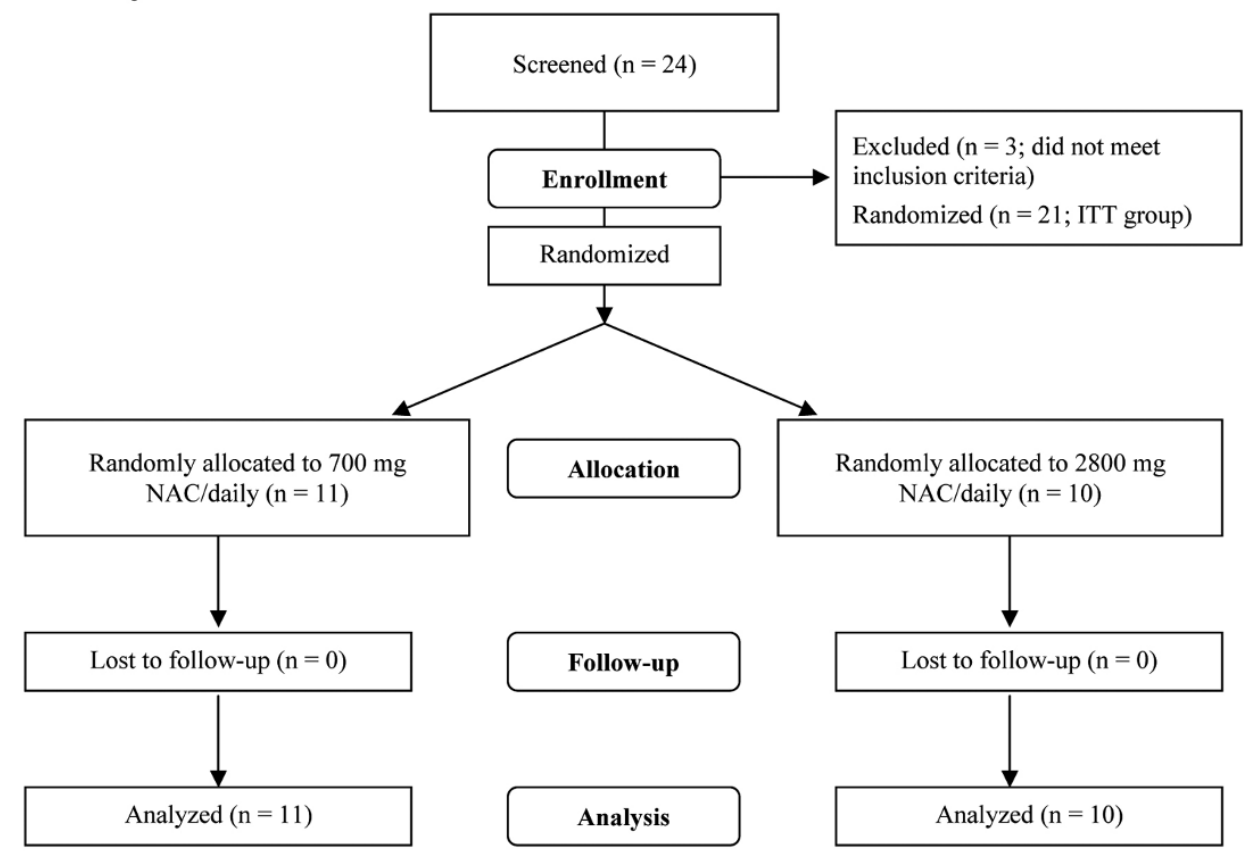

\section{B. Study design}

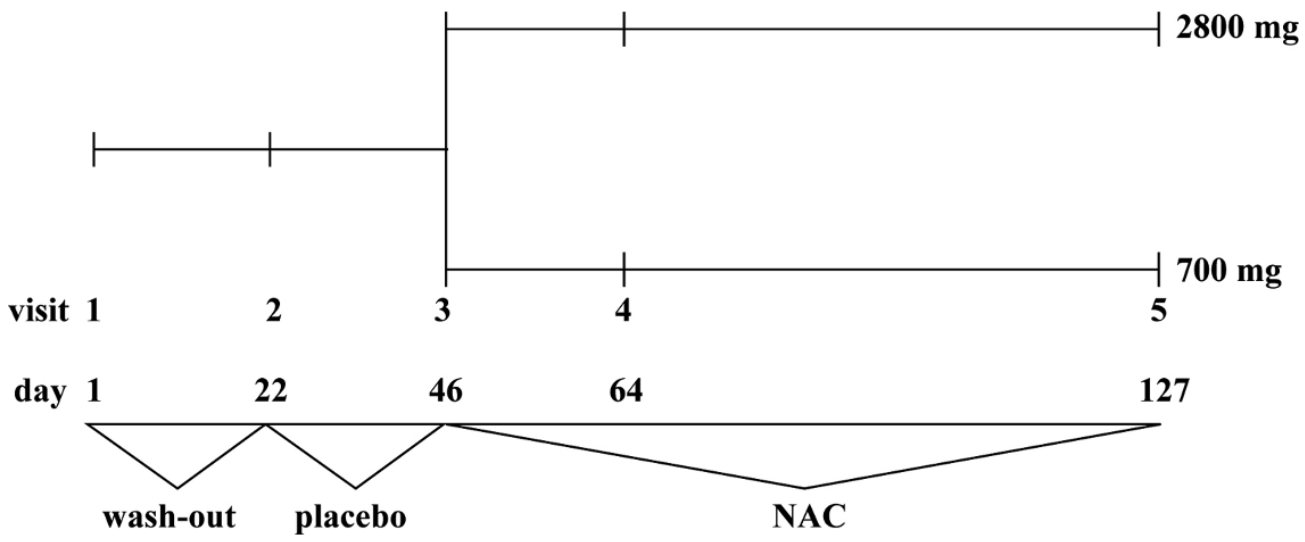

Fig. 1. A. A CONSORT study flow chart of patient enrolment, followup and analysis. B. Study design. Visit 3 (at the end of placebo run-in phase) was defined as baseline for subsequent analyses.

group phase II study with a three-week single-blind placebo run-in phase. In total, patients came 5 times to the study centre: visit 1 (screening / wash-out, day 1), visit 2 (randomisation / placebo run-in phase, day 22 ), visit 3 (begin of therapy with either $700 \mathrm{mg}$ or $2800 \mathrm{mg}$ NAC/daily, day 43), visit 4 (on study medication, day 64), and visit 5 (final visit, day 127). Patients' allocation to either dose of NAC was done according to a randomisation list (1:1 balanced) generated with Random 1.0 software. Patients were instructed to bring back empty medication containers which were used to assess patients' compliance. A patient was considered as compliant if she or he took at least $80 \%$ of the dispensed medication.

\section{SAFETy MEASures AND Adverse Events}

Safety measures comprised adverse events, changes in vital signs, and safety laboratory parameters. Adverse events were registered during visits $2,3,4$, and 5. Ex- acerbations of CF lung disease were counted as adverse events. Vital signs were registered during each visit at the study centre. Safety laboratory comprised analyses of serum (AST / ALT, $\boldsymbol{\gamma}$-GT, C-reactive protein), blood, and urine parameters. These were obtained on visit 1 and 5 . In addition, an overall tolerability was assessed by both investigator and patient at visit 5 .

\section{EfFicacy Measures}

The following parameters were quantified for the subsequent efficacy evaluation: pulmonary functions tests, antioxidant markers (extracellular glutathione in induced sputum and blood), and inflammation markers (total leukocyte counts and cell differentials, Tumour Necrosis Factor (TNF) $\alpha$, and Interleukin (IL) -8) in induced sputum. In addition, changes in disease symptoms (clinical symptoms / Quality of Life Questionnaire) were also registered during each visit. 


\section{Sputum Induction, Processing, And Outcome} MeAsures

Sputum samples were collected during visits 2, 3, 4 and 5. Sputum induction was performed according to the standard protocols as described by us previously [11]. Sputum samples were obtained, and small aliquots were sent for microbiology analysis. The remainders were processed as described in our previous publication [11]. This protocol enabled measures of both extracellular glutathione and cytokines in sputum supernatants, as well as total leukocyte numbers and cell differentials in the sputum's solid phase. In brief, induced sputum was mixed with ice-cold phosphatebuffered saline, gently mixed and vortexed, and centrifuged to separate supernatant (to analyse glutathione and cytokines) and solid phase (to analyse cells) [11]. We did not utilise dithiothreitol to obtain sputum supernatants because this reagent affects glutathione quantification in the sputum [12].

Extracellular glutathione was quantified by the standard methodology as described by us previously [11]. Cytokines (TNF- $\alpha$ and IL-8) were quantified with ELISA kits from R\&D Systems.

The solid phase was solubilised with a mixture of dithiothreitol and rhDNAse to obtain cells [11].

\section{Blood Samples and Outcome Measures}

Blood samples were obtained during visits 2, 3, 4 and 5. Samples were collected in EDTA tubes and immediately processed for glutathione [13].

\section{Data AnALYses}

Data are presented as median (25 -75\%) throughout the text and as box-whisker (min value, 25\%, median, $75 \%$, and max value) plots in the figures.

Safety and efficacy measures on treatment with either dose of NAC (visits 4 and 5) were compared to the baseline which was defined as the end of placebo run-in, i.e. visit 3. Categorical data were analysed using Fishers exact test. Ordinal or interval data were analysed using
Mann-Whitney (between-group comparisons), Wilcoxon or Friedman tests (within-group comparisons), where appropriate. Two-tailed $\mathrm{p}$ values were used, and statistical significance was defined at $\mathrm{p}<0.05$.

\section{Role of Funding Source}

The sponsor of this study (Hexal AG) participated in the study planning and study performance, but played no role in data collection, data analysis, data interpretation, or summarising the study results and writing this manuscript.

\section{RESULTS \\ SubJECTS}

A total of twenty-four patients were screened and asked to participate in the study. Twenty-one patients met the inclusion criteria and were included in the intention-to-treat analysis (Table 1). Eleven patients were randomly assigned to receive $700 \mathrm{mg} /$ daily of NAC (low-dose; Table 1), and ten patients were randomly assigned to receive $2800 \mathrm{mg} /$ daily of NAC (high-dose; Table 1). Patients in both groups were comparable in demographic and clinical parameters (Table 1). For instance, both groups were not significantly different in the degree of airway obstruction at visit 1: median (25-75\%) $\mathrm{FEV}_{1}$ of 80.60 (55.30$90.00) \%$ pred. in the $700 \mathrm{mg} /$ daily NAC vs. 65.80 $(62.90-73.40) \%$ pred. in the $2800 \mathrm{mg} /$ daily NAC (Table 1 and Fig. 2, visit $1 ; \mathrm{p}=0.43$ ).

\section{COMPLiAnce}

The compliance was found to be good, with the calculated compliance rates of $>80$ per cent for both study groups.

\section{SAFETY}

Both study groups exhibited comparable numbers of mild-to-moderate adverse effects (24 on $700 \mathrm{mg} /$ daily of NAC, and 24 on $2800 \mathrm{mg} /$ daily of NAC; $>$ > 0.5).

Table 1. Patient characteristics.

\begin{tabular}{lcc}
\hline & $\mathbf{7 0 0} \mathbf{~ m g ~ N A C}$ & $\mathbf{2 8 0 0} \mathbf{~ m g ~ N A C ~}$ \\
\hline Number of patients & 11 & 10 \\
Male/female (n) & $9 / 2$ & $7 / 3$ \\
Age & $27.0(21.0-35.0)$ & $28.5(24.0-35.0)$ \\
Height $(\mathrm{cm})$ & $168.0(163.0-178.0)$ & $174.0(168.0-178.0)$ \\
Weight $(\mathrm{kg})$ & $65.5(59.0-70.40)$ & $64.5(57.4-72.0)$ \\
Pseudomonas aeruginosa & 4 & 3 \\
Staphylococcus aureus & 1 & 1 \\
Various bacteria / saprophytic flora & 6 & 6 \\
FEV $_{1}(\%$ pred $)$ & $80.6(55.3-90.0)$ & $65.8(62.9-73.4)$ \\
FVC $(\% \text { pred })_{\text {MEF }_{25-75}(\% \text { pred })}$ & $82.6(78.4-100.4)$ & $84.8(79.6-91.2)$ \\
\hline
\end{tabular}

Data are shown as median $(25-75 \%)$ values 
$700 \mathrm{mg}$

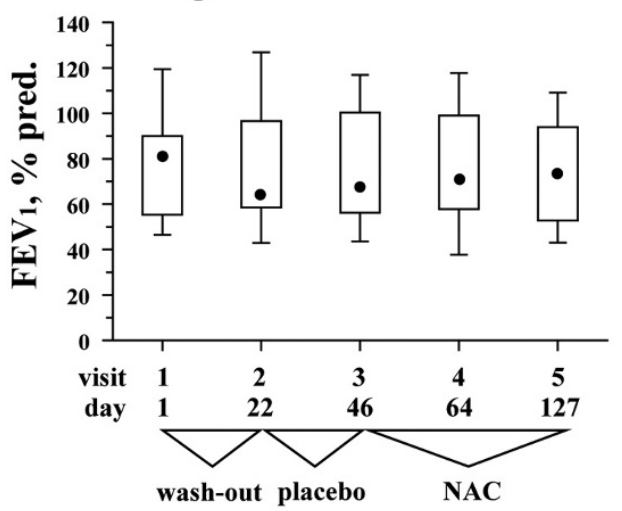

$2800 \mathrm{mg}$

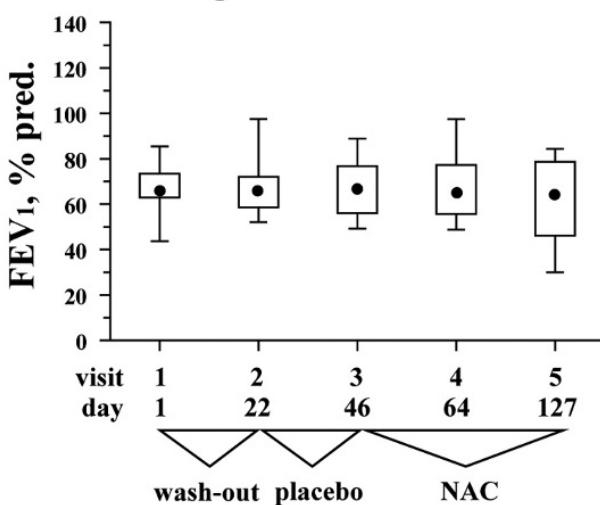

Fig. 2. Box and whisker plots for $\mathrm{FEV}_{1}$ (\% predicted) at each of the five study visits. Whiskers represent minimum and maximum values, box represents 25 and 75 percentiles, - represents median values. Visit 3 at the end of placebo run-in phase defines the baseline.

\section{A. Extracellular glutathione in induced sputum}

\section{$700 \mathrm{mg}$}

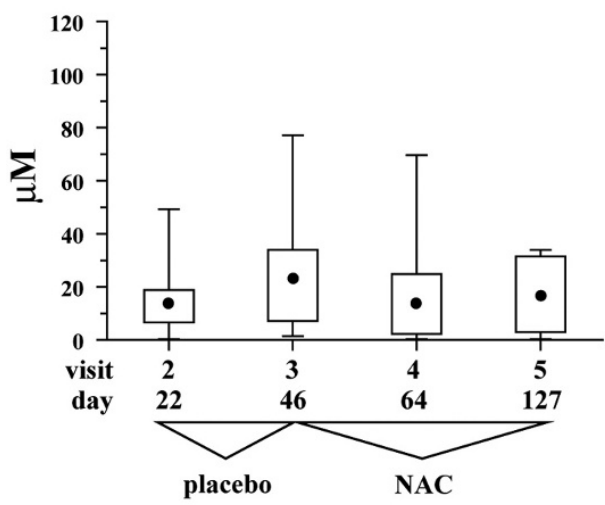

$2800 \mathrm{mg}$

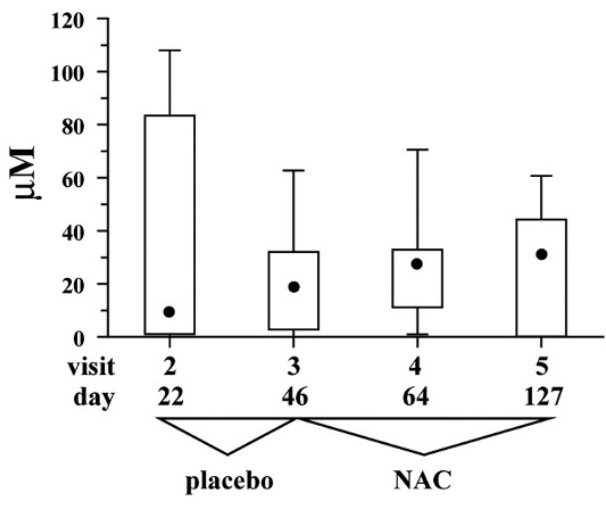

\section{B. Extracellular glutathione in blood plasma}
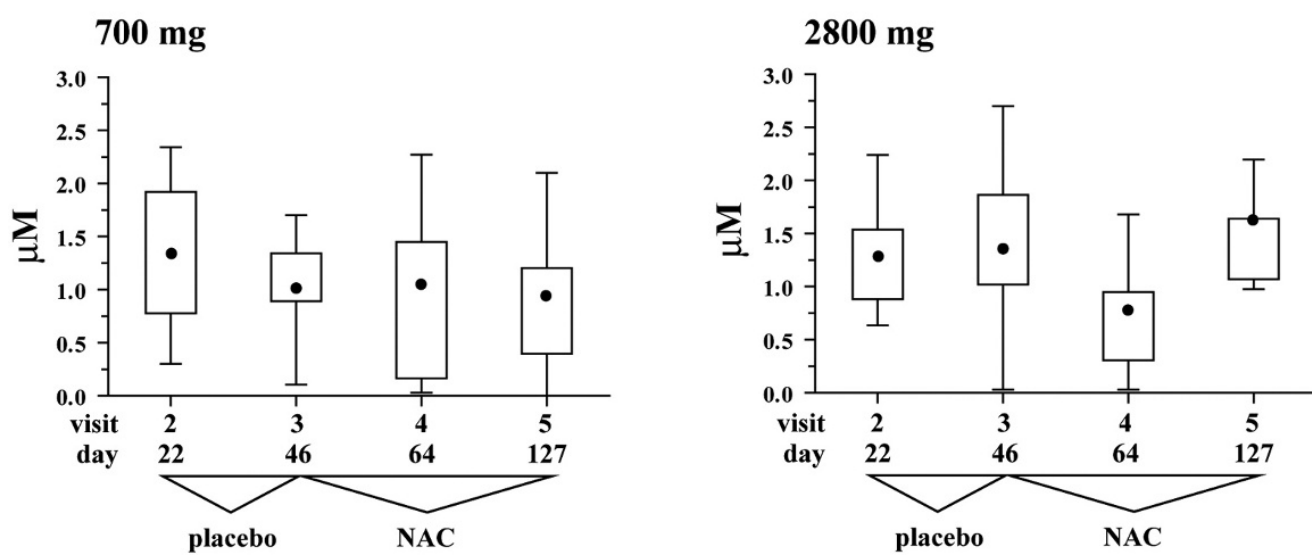

Fig. 3. A. Box and whisker plots for extracellular glutathione in induced sputum at the four study visits when induced sputum samples were collected. Whiskers represent minimum and maximum values, box represents 25 and 75 percentiles, - represents median values. Visit 3 at the end of placebo runin phase defines the baseline. B. Box and whisker plots for extracellular glutathione in blood plasma at the four study visits when blood samples were collected. Whiskers represent minimum and maximum values, box represents 25 and 75 percentiles, - represents median values. Visit 3 at the end of placebo run-in phase defines the baseline.

The adverse events were mostly exacerbations of the CF lung disease.

There have been three adverse effects which were rated as "serious". Two adverse effects (polypectomia: the $700 \mathrm{mg} /$ daily NAC group; haemoptysis during a common cold: the $2800 \mathrm{mg} \mathrm{NAC} /$ daily group) were rated as non-related to the study medication. With one adverse effect, the causal relationship between the study drug and adverse event was rated as "possible". This adverse event was in one patient in the 700 $\mathrm{mg} /$ daily NAC study group who developed gastrointestinal bleeding.
Twenty patients (both groups combined, one report missing) reported the study medication as a "very good" or "good" tolerable.

\section{EFFicacy Measures}

\section{Pulmonary function tests}

Both study groups demonstrated comparable baseline values of $\mathrm{FEV}_{1}$ before the start of NAC therapy (Fig. 2 , visit $3 ; \mathrm{p}=0.65) . \mathrm{FEV}_{1}$ did not change significantly during medication with either doses of NAC (Fig. 2; p $>0.3$ for both groups). 


\section{Extracellular total glutathione in induced sputum} and blood plasma

Both study groups demonstrated comparable concentrations of extracellular total glutathione in induced sputum at the baseline (i.e. visit 3): 21.2 (7.2 - 34.02) $\mu \mathrm{M}$ in the $700 \mathrm{mg} /$ daily NAC vs. $18.6(2.8-32.4) \mu \mathrm{M}$ in the $2800 \mathrm{mg} /$ daily NAC (Fig. 3A, visit 3; $\mathrm{p}=0.71$ ). In the $700 \mathrm{mg} /$ daily NAC, the median sputum concentrations of total glutathione tended to decrease during therapy with NAC, compared to the baseline, i.e. visit 3: $23.2(7.2-34.0) \mu \mathrm{M}$ vs. $14.0(2.3-24.8)$ at visit 4 and $16.4(3.0-31.5) \mu \mathrm{M}$ at visit 5 (Fig. $3 \mathrm{~A}$ ). However, these differences did not reach statistical significance $(\mathrm{p}>0.3)$.

On the contrary, in the $2800 \mathrm{mg} /$ daily NAC, the median values of total glutathione in induced sputum tended to increase on NAC compared to the baseline (visit 3): $18.6(2.8-32.14) \mu \mathrm{M}$ vs. $27.2(11.2-33.0)$ $\mu \mathrm{M}$ at visit 4 and $31.3(0.2-44.3) \mu \mathrm{M}$ at visit 5 (Fig. 3A). Similar to the $700 \mathrm{mg} /$ daily NAC group, these differences did not reach statistical significance (Fig. $3 \mathrm{~A}$, visits $3-5$; $\mathrm{p}>0.8$ ).

This study confirmed our previous report [11] that total glutathione in sputum samples of patients with CF is in reduced form ( $>81 \%$; data not shown).

With regard to extracellular total glutathione in blood plasma, both study groups demonstrated comparable concentrations of this antioxidant at the base- line (i.e. visit 3): $1.0(0.9-1.3) \mu \mathrm{M}$ in the $700 \mathrm{mg} /$ daily NAC vs. $1.4(1.0-1.9) \mu \mathrm{M}$ in the $2800 \mathrm{mg} /$ daily NAC (Fig. 3B, visit $3 ; \mathrm{p}=0.37$ ). In both study groups, there were no significant changes in concentrations of total glutathione in blood plasma during treatment with NAC (Fig. 3B, visits $3-5$; $\mathrm{p}>0.3$ for both groups).

\section{Inflammatory Markers in Induced Sputum}

Both groups were not significantly different in total number of leukocytes in induced sputum at the baseline (visit 3): $56.6(32.0-122.5) \times 10^{6}$ leukocytes in the $700 \mathrm{mg} /$ daily NAC vs. $31.5(20.0-113.7) \times 10^{6}$ leukocytes in the $2800 \mathrm{mg} /$ daily NAC ( $\mathrm{p}=0.51)$. In the 700 $\mathrm{mg} /$ daily NAC, total number of leukocytes did not change significantly during active therapy (visits 4 and 5: $102(55.2-164.0) \times 10^{6}$ and $56.0(36.0-235.0) \times$ $10^{6}$ leukocytes, respectively; $\mathrm{p}>0.3$ ). The same was true for the $2800 \mathrm{mg}$ /daily NAC study group (visits 4 and 5: $48.6(42.6-186.2) \times 10^{6}$ and $36.8(19.9-110.8)$ $\mathrm{x} 106$; respectively; $\mathrm{p}>0.3)$.

In both study groups, sputum leukocytes were predominantly neutrophils (data not shown).

Sputum TNF- $\alpha$. Patients in the $700 \mathrm{mg} /$ daily NAC demonstrated slightly lower concentrations of sputum TNF- $\alpha$ at the baseline (visit 3): 68.4 (30.0 - 140.0) $\mathrm{pg} / \mathrm{mL}$ vs. $140.0(105.0-541.5) \mathrm{pg} / \mathrm{mL}$; Fig. $4 \mathrm{~A} ; \mathrm{p}=$ $0.17)$. Further, in both groups TNF- $\alpha$ exhibited similar kinetics during therapy with NAC: it raised slightly

\section{A. TNF- $\alpha$ in induced sputum}

\section{$700 \mathrm{mg}$}

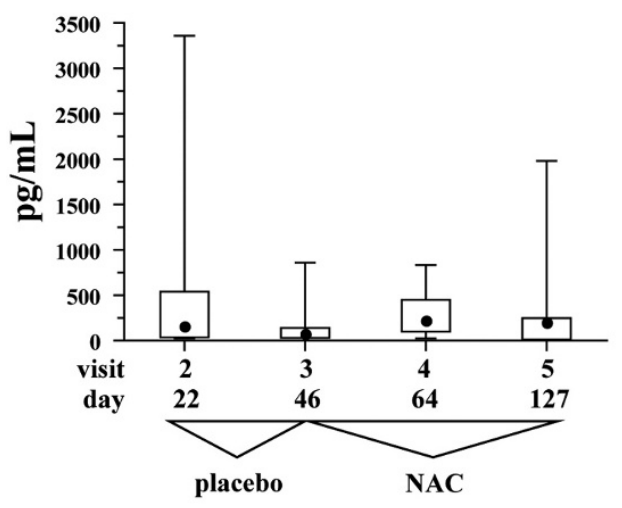

B. IL-8 in induced sputum

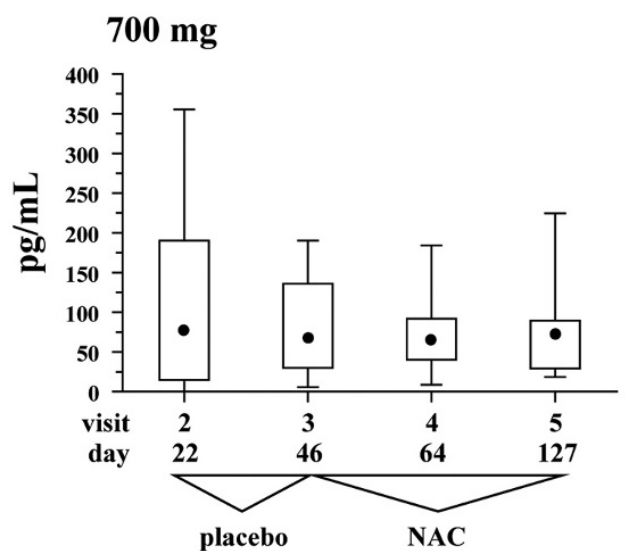

$2800 \mathrm{mg}$
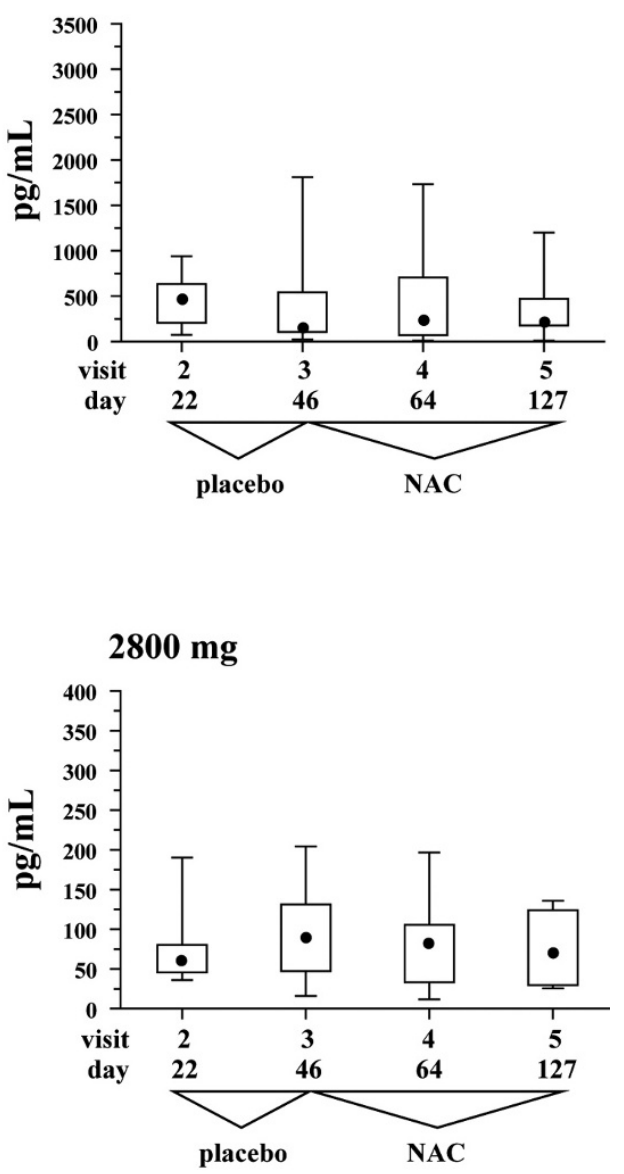

Fig. 4. A. Box and whisker plots for TNF$\alpha$ in induced sputum at the four study visits when induced sputum samples were collected. Whiskers represent minimum and maximum values, box represents 25 and 75 percentiles, - represents median values. Visit 3 at the end of placebo run-in phase defines the baseline. B. Box and whisker plots for IL-8 in induced sputum at the four study visits when induced sputum samples were collected. Whiskers represent minimum and maximum values, box represents 25 and 75 percentiles, - represents median values. Visit 3 at the end of placebo runin phase defines the baseline. 
at visit 4 and decreased at visit 5 (Fig. 4A). However, these variations did not reach statistical significance in either study group (Fig. 4A; p > 0.6 for both groups).

Sputum IL-8. Patients from both study groups demonstrated comparable concentrations of sputum IL-8. In specific, patients in the $700 \mathrm{mg} /$ daily NAC study group had $68.6(30.0-136.1) \mathrm{pg} / \mathrm{mL}$, whereas patients in the $2800 \mathrm{mg} /$ daily NAC study group demonstrated $90.2(47.4-131.2) \mathrm{pg} / \mathrm{mL}$ (Fig. 4B, visit $3 ; \mathrm{p}=0.5)$. In contrast to TNF- $\alpha$, concentrations of IL-8 in induced sputum demonstrated less variations in both study groups during therapy with NAC (Fig. $4 \mathrm{~B} ; \mathrm{p}>0.1$ for both groups).

\section{Discussion}

High-dose oral NAC is a promising new drug to suppress oxidative stress and excessive inflammation in CF airways. We conducted a single-centre, randomised, double-blinded, placebo-controlled phase II study on safety and efficacy of a prolonged (12-weeks) therapy with low- (700 mg/daily) or high-dose (2800 $\mathrm{mg}$ /daily) NAC in patients with CF.

Until recently, there was only one report from a phase I study by Tirouvanziam et al [10] on high-dose NAC for therapy of CF. We were unaware of their study when the present study was designed and conducted. Both our and the study Tirouvanziam et al [10] utilised some similar measures, as well as some different ones. Therefore, both studies provide confirmatory as well as non-redundant information about safety and efficacy of high-dose NAC in patients with CF.

The first important observation similar between our and the study by Tirouvanziam et al [10] is that highdose NAC is a safe medication for therapy of CF. In our study, $2800 \mathrm{mg} /$ daily NAC was well tolerated over the course of 12 weeks. Further, neither study revealed any specific risks associated with high-dose NAC in patients with CF.

Second, neither study observed any changes in pulmonary function on high-dose NAC. Yet, it is probably premature to rule out any beneficial effects of high-dose NAC. The timeframes of both studies could have been too short for this outcome measure to show significant changes. The timeframe of our study (12 weeks) was longer, however, it may take months to detect these changes. Indeed, the studies on e.g. high-dose ibuprofen, another anti-inflammatory drug for therapy of CF, went on for 4 or 2 years $[3,4]$ to register a significant impact of the anti-inflammatory drug.

Both studies on high-dose NAC quantified glutathione, important antioxidant in $\mathrm{CF}$, which is expected to be augmented by NAC supplementation. With respect to glutathione, both studies deliver non-redundant information.

Our study focused on extracellular glutathione in induced sputum and blood plasma. Induced sputum samples airway secretions in a non-invasive way, and our research group was the first one to adapt the protocol of induced sputum processing to quantify extracellular glutathione [12], also in patients with CF [11].

The rationale to measure extracellular glutathione comes from the fact that this antioxidant might be in deficiency in CF airways [13]. This deficiency is a direct consequence of the malfunctioning glutathione-exporting function of CF transmembrane conductance regulator (CFTR) protein [14-16]. Decrease in extracellular glutathione is thought to aggravate oxidative stress and promote inflammation in CF airways [17, 18]. Therefore, therapeutic interventions capable of increasing extracellular glutathione in CF airways are expected to bring about beneficial clinical effects. Our study demonstrated that high-dose NAC tended to increase concentrations of extracellular glutathione in induced sputum. Further, these trends appeared to be specific to the airways, as no such trends were seen with regard to extracellular glutathione in blood plasma.

By contrast, Tirouvanziam et al [10] focused on studying intracellular glutathione in blood neutrophils and whole blood lysates (predominantly, red blood cells). These authors observed significant changes in intracellular glutathione in neutrophils and in whole blood lysates. It is plausible that blood cells (including neutrophils) increase their intracellular glutathione concentrations on high-dose NAC. Blood cells are likely to be exposed to the much higher concentrations of NAC than the cells in the lung.

The increase of intracellular glutathione in neutrophils could explain the trends towards increased extracellular glutathione in induced sputum samples. Previously, we reported high concentrations of reduced glutathione in sputum samples from patients with CF [11], and the present study confirmed our previous observation. Given the impairment of glutathione-exporting function of mutated CFTR in CF airway epithelium [14-16], high concentrations of reduced glutathione can be explained by its efflux from apoptotic cells, such as neutrophils. Such efflux of reduced glutathione has been shown with respect to many cell types undergoing apoptosis [19-21]. This efflux involves multidrug resistance-associated protein (MRP)$1[22,23]$, i.e. is CFTR-independent. One could speculate that high-dose NAC provides peripheral neutrophils with higher intracellular glutathione, and that these neutrophils are capable of releasing higher, than normally, concentrations of glutathione in CF airways.

Both studies studied markers of airway inflammation in induced sputum (ours: TNF- $\alpha$ and IL-8, Tirouvanziam et al: neutrophil elastase and IL-8). Both studies should be comparable because of similar methodologies to obtain sputum supernatant. Sputum supernatants were extracted with phosphate-buffered saline, i.e. without dithiothreitol. Dithiothreitol interferes with quantification of glutathione in induced sputum [12] and confounds cell functional outcomes [10].

In neither study were the induced sputum cytokines affected by high-dose NAC. Specifically, the sputum concentrations of TNF- $\alpha$ in the present study were not decreased by high-dose NAC. Further, the sputum IL-8 concentrations also remained unaffected by NAC. Supporting these data, not all patients in the study by Tirouvanziam et al [10] responded to highdose NAC by decreasing sputum IL-8. By contrast, decreases in activity of neutrophil elastase after 4 weeks on high-dose NAC were by far more prominent and significant [10]. Neutrophil elastase is a neutrophilspecific factor. Its suppression caused by high-dose 
NAC [10] may indicate that neutrophils are the primary target of this drug in patient with $\mathrm{CF}$.

There were some differences between our study and the study by Tirouvanziam et al [10]. For instance, we did not observe any significant changes in total leukocyte numbers in induced sputum, whereas they reported decrease in these numbers on high-dose NAC. These differences can be explained by the differences in lengths between the two studies (12 weeks vs. 4 weeks). The total leukocyte number is sputum varies depending on the phase of CF lung disease (remission vs. exacerbation). A prolonged 12 -weeks study is more likely to be affected by clinical exacerbations.

In conclusion, our study demonstrates that highdose NAC is a well-tolerated and safe medication for a prolonged therapy of patients with $\mathrm{CF}$ with a potential capacity to increase extracellular glutathione in $\mathrm{CF}$ airways. Long-term and larger studies are necessary to confirm the antioxidant and anti-inflammatory activity of high-dose NAC, and its implications on clinical disease in patients with $\mathrm{CF}$.

Acknowledgements: The authors would like to thank all the patients who participated in this study. The authors further thank Mrs. Rafaela Paxinos, Mrs. Ulrike Müller, Dr. Christian von Mallinckrodt and Dr. Tim Hirche for their invaluable help with the present study.

The study was supported by Hexal AG, Holzkirchen, Germany.

\section{REFERENCES}

1. Eigen H, Rosenstein BJ, FitzSimmons S, Schidlow DV. A multicenter study of alternate-day prednisone therapy in patients with cystic fibrosis. Cystic Fibrosis Foundation Prednisone Trial Group. J Pediatr. 1995 Apr;126(4):51523.

2. Auerbach HS, Williams M, Kirkpatrick JA, Colten HR. Alternate-day prednisone reduces morbidity and improves pulmonary function in cystic fibrosis. Lancet. 1985 Sep 28;2(8457):686-8.

3. Konstan MW, Byard PJ, Hoppel CL, Davis PB. Effect of high-dose ibuprofen in patients with cystic fibrosis. N Engl J Med. 1995 Mar 30;332 (13):848-54.

4. Lands LC, Milner R, Cantin AM, Manson D, Corey M. High-dose ibuprofen in cystic fibrosis: Canadian safety and effectiveness trial. J Pediatr. 2007 Sep;151(3):249-54.

5. Lai HC, FitzSimmons SC, Allen DB et al. Risk of persistent growth impairment after alternate-day prednisone treatment in children with cystic fibrosis. N Engl J Med. 2000 Mar 23;342(12):851-9.

6. Chmiel JF, Konstan MW. Inflammation and anti-inflammatory therapies for cystic fibrosis. Clin Chest Med. 2007 Jun;28(2):331-46.

7. Schwarzer C, Fischer H, Kim EJ et al. Oxidative stress caused by pyocyanin impairs CFTR $\mathrm{Cl}(-)$ transport in human bronchial epithelial cells. Free Radic Biol Med. 2008 Dec 15;45(12):1653-62.

8. Rada B, Lekstrom K, Damian S, Dupuy C, Leto TL. The Pseudomonas toxin pyocyanin inhibits the dual oxidasebased antimicrobial system as it imposes oxidative stress on airway epithelial cells. J Immunol. 2008 Oct 1;181(7): 4883-93.

9. Witko-Sarsat V, Delacourt C, Rabier D, Bardet J, Nguyen AT, Descamps-Latscha B. Neutrophil-derived long-lived oxidants in cystic fibrosis sputum. Am J Respir Crit Care Med. 1995 Dec;152(6 Pt 1):1910-6.
10. Tirouvanziam R, Conrad CK, Bottiglieri T, Herzenberg LA, Moss RB, Herzenberg LA. High-dose oral N-acetylcysteine, a glutathione prodrug, modulates inflammation in cystic fibrosis. Proc Natl Acad Sci U S A. 2006 Mar 21;103(12):4628-33.

11. Dauletbaev N, Viel K, Buhl R, Wagner TO, Bargon J. Glutathione and glutathione peroxidase in sputum samples of adult patients with cystic fibrosis. J Cyst Fibros. 2004 Jun;3(2):119-124.

12. Dauletbaev N, Rickmann J, Viel K, Buhl R, Wagner TO, Bargon J. Glutathione in induced sputum of healthy individuals and patients with asthma. Thorax. 2001 Jan;56(1):13-8.

13. Roum JH, Buhl R, McElvaney NG, Borok Z, Crystal RG. Systemic deficiency of glutathione in cystic fibrosis. J Appl Physiol. 1993 Dec;75(6):2419-24.

14. Kogan I, Ramjeesingh M, Li C et al. CFTR directly mediates nucleotide-regulated glutathione flux. EMBO J. 2003 May 1;22(9):1981-9.

15. Gao L, Kim KJ, Yankaskas JR, Forman HJ. Abnormal glutathione transport in cystic fibrosis airway epithelia. Am J Physiol. 1999 Jul;277(1 Pt 1):L113-8.

16. Linsdell P, Hanrahan JW. Glutathione permeability of CFTR. Am J Physiol. 1998 Jul;275(1 Pt 1):C323-6.

17. Terheggen-Lagro SW, Rijkers GT, van der Ent CK. The role of airway epithelium and blood neutrophils in the inflammatory response in cystic fibrosis. J Cyst Fibros. 2005 Aug;4 Suppl 2:15-23.

18. Jacquot J, Tabary O, Le RP, Clement A. Airway epithelial cell inflammatory signalling in cystic fibrosis. Int J Biochem Cell Biol. 2008;40(9):1703-15.

19. Ghibelli L, Coppola S, Rotilio G, Lafavia E, Maresca V, Ciriolo MR. Non-oxidative loss of glutathione in apoptosis via GSH extrusion. Biochem Biophys Res Commun. 1995 Nov 2;216(1):313-20.

20. van den Dobbelsteen DJ, Nobel CS, Schlegel J, Cotgreave IA, Orrenius S, Slater AF. Rapid and specific efflux of reduced glutathione during apoptosis induced by antiFas/APO-1 antibody. J Biol Chem. 1996 Jun 28;271(1): 15420-7.

21. Hammond CL, Madejczyk MS, Ballatori N. Activation of plasma membrane reduced glutathione transport in death receptor apoptosis of HepG2 cells. Toxicol Appl Pharmacol. 2004 Feb 15;195(1):12-22.

22. Hammond CL, Marchan R, Krance SM, Ballatori N. Glutathione export during apoptosis requires functional multidrug resistance-associated proteins. J Biol Chem. 2007 May 11;282(19):14337-47.

23. Marchan R, Hammond CL, Ballatori N. Multidrug resistance-associated protein 1 as a major mediator of basal and apoptotic glutathione release. Biochim Biophys Acta. 2008 Oct;1778(10):2413-20.

Received: May 14, 2009 / Accepted: June 5, 2009

Address for correspondence:

Prof. Joachim Bargon

Hospital St. Elisabeth

Ginnheimer Str. 3

60487 Frankfurt/Main

Germany

Tel.: $\quad+496979392119$

Fax: + 496979392669

E-mail: bargon@em.uni-frankfurt.de 